\section{OC-059 PREVALENCE OF BILE ACID MALABSORPTION AS A CAUSE OF DIARRHOEA IN CONSECUTIVE NEW PATIENT REFERRALS TO A GASTROENTEROLOGY CLINIC}

doi:10.1136/gutjpl-2013-304907.058

1."U Shivaji, ${ }^{2} \mathrm{~F}$ Chowdhury, ${ }^{1} \mathrm{~A}$ C Ford. ' Gastroenterology; ${ }^{2}$ Nuclear Medicine, Leeds Teaching Hospitals, Leeds, UK

Introduction Background Diarrhoea is a common presenting complaint in the Gastroenterology outpatient department. The potential causes are numerous, but include irritable bowel syndrome (IBS), inflammatory bowel disease (IBD), coeliac disease, and colorectal cancer. Interest in bile acid malabsorption (BAM) as a cause of diarrhoea has increased recently. However, guidelines from the British Society of Gastroenterology do not recommend routine exclusion of this condition using 23-seleno-25-homo-tauro-cholic acid (SeHCAT) scanning.

Methods Review of consecutive unselected new patient referrals to a single Gastroenterologists' outpatient clinic during a 2-year period, from January 2010 to December 2011. All clinic letters were reviewed retrospectively, and symptoms reported by the patient at the initial consultation were recorded. Radiology, endoscopy, chemical pathology, and histopathology databases were then cross-examined in order to ascertain the final diagnosis following full investigation, to the level deemed appropriate by the consulting physician. We defined BAM using a SeHCAT retention value of $<15 \%$ at 7 days.

Results Of 397 consecutive unselected new patient referrals to a single Gastroenterologist between January 2010 and December 2011, 102 (25.7\%) reported diarrhoea. After investigation the final diagnoses are listed in Table 1 . The second commonest cause of diarrhoea, after IBS, was BAM. Eight (53.3\%) of 15 patients with BAM reported lower abdominal pain or discomfort. In 10 (66.7\%) patients there was no obvious cause of BAM, and these were classified as idiopathic, or type II, BAM.

\section{Abstract 0C-059 Table 1}

\begin{tabular}{lll}
\hline & Number $(\mathbf{n}=\mathbf{1 0 2})$ & Percentage \\
\hline IBS & 27 & 26.5 \\
BAM & 15 & 14.7 \\
IBD & 12 & 11.8 \\
Functional diarrhoea & 7 & 6.9 \\
Coeliac & 5 & 4.9 \\
PPI-related & 5 & 4.9 \\
Collagenous colitis & 4 & 3.9 \\
Pancreatic insufficiency & 2 & 2.0 \\
Colorectal cancer & 1 & 1.0 \\
\hline
\end{tabular}

Conclusion BAM was the commonest underlying cause of diarrhoea after IBS. Idiopathic BAM was commoner than coeliac disease. More than $50 \%$ of patients with BAM reported lower abdominal pain or discomfort, which may lead to misdiagnosis as IBS unless further investigations are performed. BAM should be considered as a likely diagnosis in all patients with diarrhoea, and SeHCAT scanning should be moved up the hierarchy of diagnostic tests in such patients.

Disclosure of Interest None Declared

\section{OC-060 DOES IGA TISSUE TRANSGLUTAMINASE ANTIBODY LEVELS CORRELATE WITH HISTOLOGICAL FINDINGS OF COELIAC DISEASE (CD)?}

doi:10.1136/gutjnl-2013-304907.059

1."M Kurien, 'A J Johnston, 'A Avgerinos, 'D S Sanders. 'Department of Gastroenterology, Royal Hallamshire Hospital, Sheffield, UK
Introduction The current gold standard diagnostic test for CD is oesophogastroduodenoscopy (OGD) and duodenal biopsies, aiming to demonstrate the presence of villous atrophy. Given the low sensitivity of endoscopic markers and the patchy distribution of $\mathrm{CD}$ within the small bowel, a duodenal bulb biopsy is currently recommended. However, recent studies have raised questions about the need for biopsies in $\mathrm{CD}$, particularly within the paedatric literature, with high anti-tissue transglutaminase antibody (TTG) levels believed to be sufficient. The hypothesis being that high TTG levels correlate closely with definitive CD histology (Marsh 3a-c) giving a high positive predicative value (PPV). This study evaluates TTG levels and histology (inclusive of a bulb biopsy) in adult patients suspected of having $\mathrm{CD}$, with the aim of defining a cut off value for TTG when biopsy may be unnecessary.

Methods Recruitment occurred between Nov 2008 and July 2012 523 adult patients ( $>16$ years). All patients had a minimum of 5 duodenal biopsies taken whilst on a gluten containing diet. Furthermore, all patients were tested for IgA TTG, Endomysial Antibody (EMA) and total IgA immunoglobulin at the time of their endoscopy. This study retrospectively reviews the correlation between TTG levels (Aesku Diagnostics, Wendelsheim, Germany) and histological outcomes in these patients, with CD being defined as villous atrophy (Marsh $3 \mathrm{a}-3 \mathrm{c}$ ) and a clinical and serological response to a gluten free diet.

Results Of the 523 adult patients (median age 51 years, range 16-91) recruited, $212(41 \%)$ had positive TTG serology (>15 U/ $\mathrm{ml}$ ). EMA positivity was identified in $31 \%$ of the cohort $(163 / 523)$ with CD diagnosed in 32\% (169/523). The sensitivity, specificity, PPV and NPV for TTG was 93.3\%, 83.3\%, 71.7\%, 96.4\% respectively and for EMA 93.8\%, 98.9\%, 97.4\% 97.2\%. Table 1 shows the PPV for diagnosing CD at differing TTG levels. No cut off level was associated with a PPV of $100 \%$, with the highest PPV value of $97.1 \%$ seen when the TTG level was set at $300 \mathrm{U} / \mathrm{ml}(20 \times$ upper normal limit).

Abstract 0C-060 Table 1 PPV for differing cut off levels for TTG

\begin{tabular}{lllll}
\hline & & \multicolumn{2}{l}{ Number of Patients } & \\
\cline { 3 - 4 } TTG cut off U/ml & $\mathbf{x}$ ULN & Coeliac disease & Not Coeliac Disease & PPV (\%) \\
\hline$>15$ & 1 & 152 & 60 & 71.7 \\
$>30$ & 2 & 146 & 34 & 81.1 \\
$>75$ & 5 & 132 & 12 & 91.7 \\
$>105$ & 7 & 128 & 9 & 93.4 \\
$>150$ & 10 & 117 & 7 & 94.4 \\
$>225$ & 15 & 105 & 5 & 95.5 \\
$>300$ & 20 & 99 & 3 & 97.1 \\
\hline
\end{tabular}

TTG; anti-tissue transglutaminase antibody; UNL; Upper Normal Limit; PPV; Positive Predicitve Value

Conclusion Contrary to ESPHGAN guidelines our findings would not support a biopsy avoidance strategy in those with high TTG levels, with patient potentially being wrongly diagnosed. We advocate that duodenal biopsies including a bulb biopsy remain the gold standard diagnostic test in those with suspected CD.

Disclosure of Interest None Declared

\section{OC-061 LONG-TERM SURVIVAL OF PATIENTS WITH SYSTEMIC SCLEROSIS ON HOME PARENTERAL NUTRITION: 27-YEARS EXPERIENCE}

doi:10.1136/gutjnl-2013-304907.060

1,2, E Harrison, 'A L Herrick, ' J McLaughlin, ${ }^{1,2}$ S Lal. 'Institute of Inflammation and Repair, FMHS, University of Manchester; ${ }^{2}$ Intestinal Failure Unit, Salford Royal NHS Foundation Trust, Salford, UK

Introduction Patients with systemic sclerosis (SSc) may develop significant gastrointestinal involvement (GI) with associated nutritional impairment. When severe, this can progress to intestinal failure (IF) 